\title{
Integrated computational biology analysis to evaluate target genes for chronic myelogenous leukemia
}

\author{
YU ZHENG ${ }^{1,2}$, YU-PING WANG ${ }^{3}, \mathrm{HONGBAO} \mathrm{CAO}^{4}$, QIUSHENG CHEN $^{1,2}$ and XI ZHANG ${ }^{2}$ \\ ${ }^{1}$ State Key Laboratory of Medical Genomics; ${ }^{2}$ Department of Hematology, \\ Ruijin Hospital Affiliated to Shanghai Jiao Tong University School of Medicine, Shanghai 200025, P.R. China; \\ ${ }^{3}$ Department of Biomedical Engineering, Tulane University, New Orleans, LA 70118; \\ ${ }^{4}$ Department of Biology Products, Life Science Solutions, Elsevier, Inc., Rockville, MD 20852, USA
}

Received December 16, 2017; Accepted April 12, 2018

DOI: $10.3892 / \mathrm{mmr} .2018 .9125$

\begin{abstract}
Although hundreds of genes have been linked to chronic myelogenous leukemia (CML), many of the results lack reproducibility. In the present study, data across multiple modalities were integrated to evaluate $579 \mathrm{CML}$ candidate genes, including literature-based CML-gene relation data, Gene Expression Omnibus RNA expression data and pathway-based gene-gene interaction data. The expression data included samples from 76 patients with CML and 73 healthy controls. For each target gene, four metrics were proposed and tested with case/control classification. The effectiveness of the four metrics presented was demonstrated by the high classification accuracy $\left(94.63 \% ; \mathrm{P}<2 \times 10^{-4}\right)$. Cross metric analysis suggested nine top candidate genes for CML: Epidermal growth factor receptor, tumor protein $\mathrm{p} 53$, catenin $\beta 1$, janus kinase 2 , tumor necrosis factor, abelson murine leukemia viral oncogene homolog 1, vascular endothelial growth factor A, B-cell lymphoma 2 and proto-oncogene tyrosine-protein kinase. In addition, 145 CML candidate pathways enriched with 485 out of 579 genes were identified $\left(\mathrm{P}<8.2 \times 10^{-11} ; \mathrm{q}=0.005\right)$. In conclusion, weighted genetic networks generated using computational biology may be complementary to biological experiments for the evaluation of known or novel CML target genes.
\end{abstract}

\section{Introduction}

Chronic myelogenous leukemia (CML) is a type of leukemia characterized by an accumulation of myeloid cells in patient blood and is caused by the uncontrolled growth of myeloid cells in the bone marrow (1). CML has a slight male

Correspondence to: Dr Qiusheng Chen, Department of Hematology, Ruijin Hospital Affiliated to Shanghai Jiao Tong University School of Medicine, 197 Ruijin Er Road, Shanghai 200025, P.R. China

E-mail: cqs11160@rjh.com.cn

Key words: chronic myelogenous leukemia, enrichment analysis, gene-gene interaction network predominance (1) and people with a median age of 65-years are more susceptible to the disease (2). Although the cause of most CML cases remains unknown, it has been suggested that genetic variations may serve a role in the pathogenesis of CML (3).

To date, hundreds of genes have been linked to CML. For example, the fusion gene breakpoint cluster region-Abelson murine leukemia viral oncogene homolog 1 (BCR-ABL1) leads to increased expression of ABL1 and has been observed in many cases of CML (4-6). ABL1 inhibitors have been developed to inhibit tyrosine kinase activity and are often used to treat patients with CML. In addition, serum levels of interleukin (IL)-6 and tumor necrosis factor (TNF) have been suggested as potential prognostic markers for $\operatorname{CML}(7,8)$. Other genes, including ephrin type-B receptor 4 , janus kinase 2 (JAK2), epidermal growth factor receptor (EGFR), catenin $\beta 1$ (CTNNB1), vascular endothelial growth factor A (VEGFA), KIT proto-oncogene receptor tyrosine kinase and tumor protein p53 (TP53), have been suggested to be significantly linked to CML, albeit with unknown mechanisms (9-14). Nevertheless, many of the genes reported to be associated with CML have been reported by very few studies, and these studies have largely been conducted with small sample sizes. Therefore, there is an increased requirement to systematically evaluate these CML target genes.

Over the last few years, Pathway Studio ${ }^{\circledR}$ (PS; www.pathwaystudio.com) has become a popular tool for literature-based relation data analysis (15-17). In the present study, PS was used to identify CML target genes for evaluation, based on published scientific literature. Multiple levels of analysis between these target genes and CML were conducted by integrating data across different modalities. The present study aimed to provide a systematic evaluation approach through a weighted gene-gene interaction (GGI) network analysis, in order to better understand the underlying pathogenic development of CML.

\section{Materials and methods}

Study design. Large-scale CML-gene relation data were analyzed to identify CML candidate genes and generate two literature-based metrics (i.e., reference and age scores). 
Subsequently, multiple analyses of target genes and CML were conducted to identify CML candidate pathways, build GGI networks and develop two network metrics (i.e., pathway and significance scores). The analysis included GGI analysis, sub-network enrichment analysis (SNEA) and gene set enrichment analysis (GSEA). Lastly, a Euclidean distance-based classification approach (18) was employed to test the effectiveness of the proposed metrics using gene expression data.

Using the aforementioned approach, a genetic database for CML (CML_GD) was developed (http://gousinfo. com/database/Data_Genetic/CML_GD.xlsx). The database contained $579 \mathrm{CML}$ target genes (CML_GD $\rightarrow$ Related Genes), supported by 1,739 references (CML_GD $\rightarrow$ Ref for Disease-Gene Relation). The database also included 145 pathways from GSEA (CML_GD $\rightarrow$ Related Pathways) and 99 diseases from SNEA (CML_GD $\rightarrow$ Related Diseases). Further information regarding the database can be found at $(\mathrm{CML}$ GD $\rightarrow$ Database Note).

CML target genes. The 579 CML target genes (CML_ $\mathrm{GD} \rightarrow$ Related Genes) were genes that had been associated with CML once or several times within PS. PS identified CML-gene relations using a natural language processing technique, covering a large literature database with $>35$ million references $(19,20)$.

Enrichment analysis. Using the 579 CML target genes as input, GSEA and SNEA were conducted within PS. GSEA compared the $579 \mathrm{CML}$ target genes against the Gene Ontology (GO) terms, PS Ontology and >2,000 PS-curated pathways. SNEA, another PS built-in enrichment module (21), identified that the 579 target genes significantly overlapped with genes associated with diseases. P-values were calculated using the Fisher's exact test, with false discovery rate (FDR) analysis for multiple analyses (22). The Fisher's exact test has been adopted as an effective statistical method to measure the gene-enrichment in annotation terms. Subsequently, GGI analysis was conducted to generate a weighted GGI network. The number of pathways shared by two genes was used as the weight for the corresponding edge.

Four network metrics. For each of the 579 CML target genes four metrics were proposed. The reference sore (RScore) of a gene was defined as the number of references supporting the CML-gene relation, according to the following equation: $\mathrm{RScore}=$ number of references supporting a CML-gene relation. The age score (AScore) of a gene was defined as the age of the reference that first reported the CML-gene relation. The pathway score (PScore) was defined as the number of pathways in CML_GD $\rightarrow$ Related Pathways that included the gene of interest. The network significance score (SScore) of a node/gene was defined as follows:

$$
\text { SScore }=\frac{N \times C_{D}^{W_{0.5}}(\mathrm{i})}{\sum_{j}^{N} C_{D}^{W_{0.5}}(\mathrm{i})}
$$

where $N$ represents the CML target gene number in the network and $C_{D}^{W 0.5}$ is the generalized centrality of the ith node. A detailed calculation of $C_{D}^{W 0.5}$ is described in previous studies (23-25).
Validation by case/control classification. R 3.1.2 software was used for the analysis. Based on the hypothesis that an effective metric could be used to select genes that were more relevant to the disease, the present study proposed to use gene expression of the top genes ranked by different metrics to classify CML cases from controls. A Euclidean distance-based classification approach (26) was employed as a classifier, followed by a leave-one-out (LOO) cross-validation. The Euclidean distance-based classification approach has been successfully applied to the classification of functional magnetic resonance imaging data as well as single nucleotide polymorphisms data in previous studies $(18,26)$. The input of the classifier was the gene expression of selected genes. For each run of the LOO processes, gene expression values of one sample were used as a testing sample and the remainder as training samples. The output was the classification accuracy (CR), which was defined as the number of correctly classified subjects of the total subjects. Lastly, a 5,000-run permutation was performed to generate a permutation P-value, which stated the probability a randomly selected gene set containing the same number of genes could reach the same or higher $\mathrm{CR}$.

The expression data used for case/control classification were acquired from 149 subjects, including samples from 76 patients with CML and 73 healthy controls (NCBI GEO: GSE13159; https:/www.ncbi.nlm.nih.gov/geo/). The dataset included 549 out of 579 CML target genes (CML_GD $\rightarrow$ Related Genes).

\section{Results}

CML target genes. A total of $579 \mathrm{CML}$ candidate genes were identified from CML-gene relation data supported by 1,739 references (Fig. 1). These genes are presented in CML_GD $\rightarrow$ Related Genes. The 1,739 supporting references are presented in CML_GD $\rightarrow$ Ref for Disease-Gene Relation, which included types of CML-gene relations, reference titles and the reference where a disease-gene relation has been identified. In addition, Fig. 1 revealed the relations between 265 out of the 579 genes and BCR-ABL mutations. It is widely accepted that BCR-ABL mutations are significantly associated with the treatment of patients with CML (27). Therefore, the present study also aimed to investigate BCR-ABL mutation-related genes and discuss their relationship to CML. The BCR-ABL-related genes and supporting references are presented in $\mathrm{CML}$ _GD $\rightarrow \mathrm{BCR}-\mathrm{ABL}$ related Genes and CML_GD $\rightarrow$ Ref for BCR-ABL-Gene Relation, respectively.

Of the 579 CML candidate genes, 325 (56.13\%) have been reported with one reference (RScore $=1), 88(15.20 \%)$ with two, $47(8.12 \%)$ with 3 and only $65(11.23 \%)$ with more than five references. As presented in Fig. 2, these 579 CML genes demonstrated several different types of functional associations with CML. While all these studies are valuable, further evaluation with meaningful metrics may help to further evaluate the linkage between these genes and CML, and reduce possible noise introduced within individual studies.

The publication date distribution for the 1,739 supporting references was also analyzed. The results demonstrated that on average the publication age of the references was 6.4 years. In addition, novel genes were identified each year. 

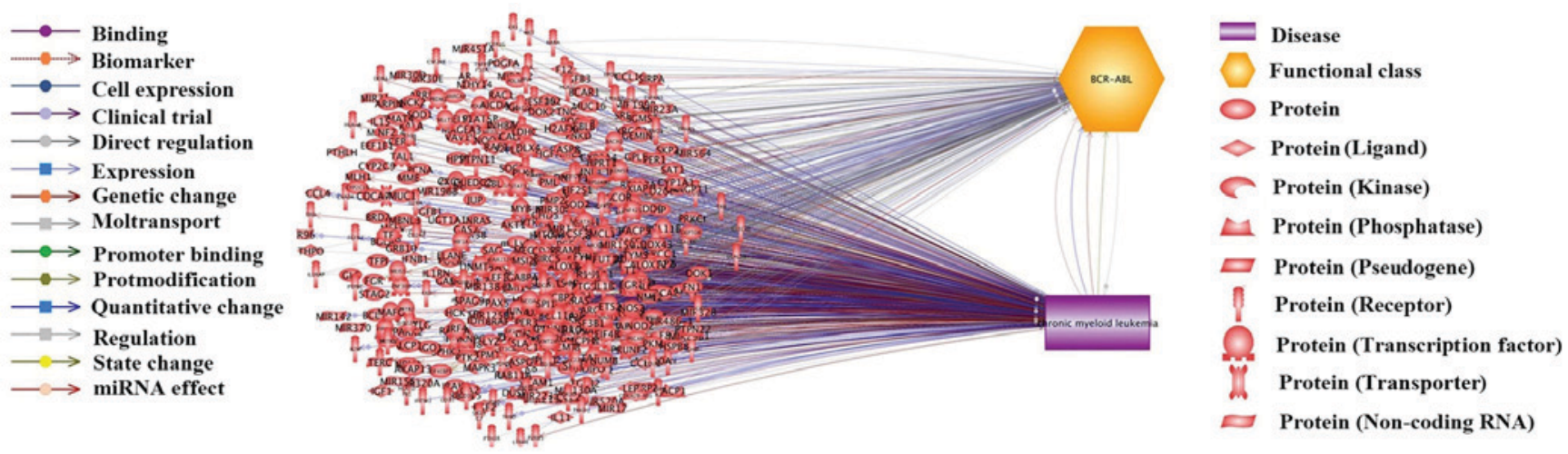

Figure 1. Relationship network between CML, BCR-ABL and the 579 CML candidate genes (proteins). The network was generated using Pathway Studio ${ }^{\circledR}$. BCR-ABL, breakpoint cluster region-Abelson murine leukemia viral oncogene homolog 1; CML, chronic myelogenous leukemia.

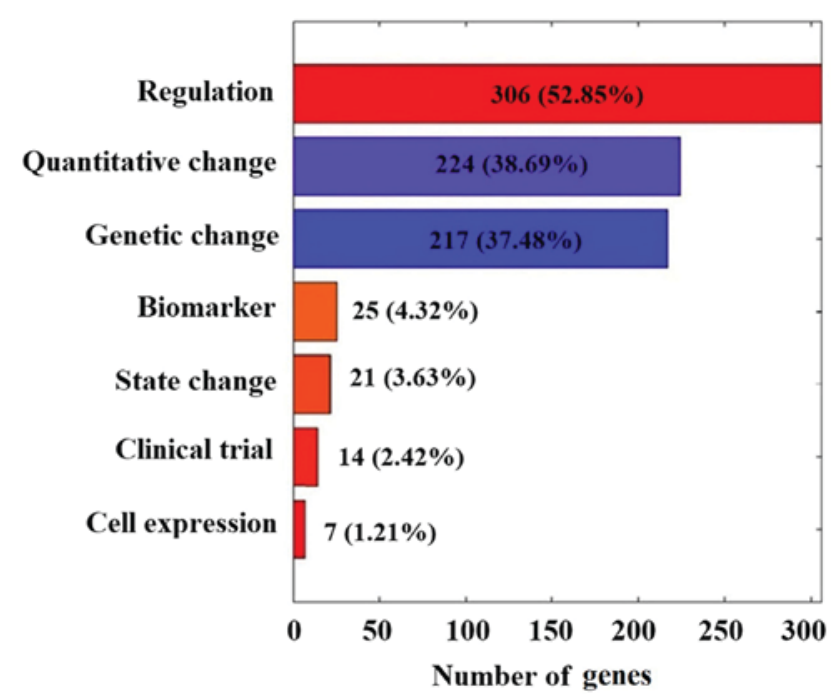

Figure 2. Various functional associations between the $579 \mathrm{CML}$ candidate genes and CML.

Enrichment analysis. The top 20 significantly enriched pathways are listed in Table I. A total of 389 out of 579 genes were enriched within these pathways $\left(\mathrm{P}<1.2 \times 10^{-27} ; \mathrm{q}=0.005\right)$. In CML_GD $\rightarrow$ Related Pathways, the top 145 pathways that were significantly enriched with 485 out of 579 genes $\left(\mathrm{P}<8.1 \times 10^{-11}\right.$; $\mathrm{q}=0.005)$ are presented.

Within the 145 pathways (CML_GD $\rightarrow$ Related Pathways), 12 were related to cell growth and proliferation (overlapped genes: 221), 11 to protein kinase (overlapped genes: 156), 11 to cell apoptosis (overlapped genes: 201), eight to protein phosphorylation (overlapped genes: 119), six to transcription factors (overlapped genes: 152), three to nervous system (overlapped genes: 89) and three to immune system (overlapped genes: 140). Additionally, two ontology terms were related to ageing (overlapped genes: 140).

Along with GSEA, SNEA was also conducted to identify the diseases that were significantly linked to the $579 \mathrm{CML}$ target genes. A total of 99 diseases are presented in CML _GD $\rightarrow$ Related Diseases and the top 10 diseases are listed in Table II. The genes associated with each of these 99 diseases demonstrated significant overlap with the 579 CML target genes $\left(\mathrm{P}<1 \times 10^{-90}\right)$.
GGI analysis. Pathway-based GGI analysis was conducted on all 579 candidate genes to build a gene interaction network for $\mathrm{CML}$, as shown in Fig. 3. The network was composed of 485 out of 579 genes and 51,072 edges. An edge between two genes represents at least one shared pathway. The remaining 94 out of 579 genes were not included, as these were not enriched within the 145 significant pathways $\left(C M L \_G D \rightarrow\right.$ Related Pathways). The adjacent matrix of the network is presented in CML_GD $\rightarrow$ GGI Network. In the adjacent matrix, the number between two genes represents the number of shared pathways. For further statistics, please refer to CML_GD $\rightarrow$ Network Statistics.

Evaluation by case/control classification. For case/control classification, the 579 target genes were initially ranked by different metrics and then the expression values of the top $n$ $(n=1,2 \ldots)$ genes were used as the input for classification. In each run of the LOO process, expression data of these input genes from one sample were used as the test, and the remainder as the training dataset for classification. The process was repeated for each sample and each $n$. The output from the LOO process was CR. The CRs obtained using a number of different genes for classification are presented in Fig. 4. Further results are provided in Table III.

Both Fig. 4 and Table III demonstrated that high CR can be achieved by using top genes ranked by different scores, whereas using more lower-scored genes may not increase the CRs (Fig. 4, the highest CR were presented at the corresponding number of genes). The results suggested that the proposed metrics were effective in selecting top genes to discriminate CML cases from controls. Notably, RScore required the smallest number of genes and had the most significant permutation $\mathrm{P}$-value $\left(\mathrm{P}<2 \times 10^{-4}\right)$, and SScore led to the highest CR of $95.30 \%$.

Cross metrics analysis. If a gene satisfied the following, it may be more likely to be associated with the development of CML: i) The gene has been supported by multiple studies for its relation with CML (high RScore); ii) the gene is involved in high number of molecular pathways implicated in CML (high PScore); and iii) the gene possesses strong connections with other CML candidate genes (high SScore). AScore presented the history of a relation between a gene and CML; the LOO 
Table I. Top 20 gene pathways/groups enriched by 389 out of 579 target genes.

\begin{tabular}{lccccc}
\hline Pathway/gene set name & GO ID & No. of entities & Overlap & $\begin{array}{c}\text { P-value } \\
\text { (FDR correction) }\end{array}$ & P-value \\
\hline Response to drug & 0017035 & 509 & 94 & $4.36 \times 10^{-51}$ & $4.36 \times 10^{-54}$ \\
Positive regulation of cell proliferation & 0008284 & 568 & 90 & $3.20 \times 10^{-43}$ & $6.39 \times 10^{-46}$ \\
Negative regulation of apoptotic process & 0006916 & 650 & 94 & $5.64 \times 10^{-42}$ & $1.69 \times 10^{-44}$ \\
Blood coagulation & 0007596 & 501 & 80 & $1.35 \times 10^{-38}$ & $5.38 \times 10^{-41}$ \\
Response to organic cyclic compound & 0014070 & 253 & 59 & $1.34 \times 10^{-37}$ & $6.69 \times 10^{-40}$ \\
Negative regulation of cell proliferation & 0008285 & 471 & 72 & $2.30 \times 10^{-33}$ & $1.38 \times 10^{-35}$ \\
Cytokine-mediated signaling pathway & 0019221 & 316 & 60 & $5.50 \times 10^{-33}$ & $3.85 \times 10^{-35}$ \\
Positive regulation of ERK1 and ERK2 cascade & 0070374 & 134 & 42 & $2.46 \times 10^{-32}$ & $1.97 \times 10^{-34}$ \\
Innate immune response & 0002226 & 792 & 90 & $5.02 \times 10^{-32}$ & $4.51 \times 10^{-34}$ \\
Regulation of cell proliferation & 0042127 & 240 & 52 & $1.59 \times 10^{-31}$ & $1.59 \times 10^{-33}$ \\
Positive regulation of apoptotic process & 0043065 & 393 & 64 & $2.33 \times 10^{-31}$ & $2.57 \times 10^{-33}$ \\
Response to hypoxia & 0001666 & 259 & 53 & $6.63 \times 10^{-3} 1$ & $7.95 \times 10^{-33}$ \\
Apoptotic process & 0008632 & 790 & 88 & $9.39 \times 10^{-31}$ & $1.22 \times 10^{-32}$ \\
Aging & 0016280 & 254 & 52 & $2.16 \times 10^{-30}$ & $3.14 \times 10^{-32}$ \\
Positive regulation of transcription, DNA-templated & 0045941 & 623 & 76 & $5.27 \times 10^{-29}$ & $8.95 \times 10^{-31}$ \\
Response to ethanol & 0017036 & 161 & 42 & $5.27 \times 10^{-29}$ & $8.96 \times 10^{-31}$ \\
Response to estradiol & 0032355 & 175 & 43 & $1.48 \times 10^{-28}$ & $2.66 \times 10^{-30}$ \\
Neurotrophin TRK receptor signaling pathway & 0048011 & 280 & 52 & $2.53 \times 10^{-28}$ & $4.81 \times 10^{-30}$ \\
Epidermal growth factor receptor signaling pathway & 0007173 & 201 & 45 & $4.43 \times 10^{-28}$ & $8.87 \times 10^{-30}$ \\
Negative regulation of transcription from RNA & 0000122 & 799 & 84 & $1.18 \times 10^{-27}$ & $2.48 \times 10^{-29}$ \\
polymerase II promoter & & & & &
\end{tabular}

The P-value for each gene set was calculated using the one-tailed Fisher's exact test against the hypothesis that a randomly selected gene set of the same size (579) could present an equal or higher number of overlapped genes with the corresponding gene set. All the gene sets passed FDR correction $(\mathrm{q}=0.005)$. FDR, false discovery rate; GO, Gene Ontology.

Table II. Sub-networks enriched by the 579 genes.

\begin{tabular}{lcccc}
\hline Gene set & Total no. of related genes & Overlap & P-value (FDR correction) & P-value \\
\hline Acute myeloid leukemia & 1,023 & 295 & $5.91 \times 10^{-256}$ & $1.77 \times 10^{-258}$ \\
Lymphoma & 967 & 254 & $7.44 \times 10^{-204}$ & $5.21 \times 10^{-206}$ \\
Myelodysplastic syndrome & 504 & 203 & $6.37 \times 10^{-202}$ & $5.10 \times 10^{-204}$ \\
Acute lymphoblastic leukemia & 582 & 212 & $1.45 \times 10^{-200}$ & $1.30 \times 10^{-202}$ \\
Non-small cell lung cancer & 1,568 & 296 & $2.41 \times 10^{-198}$ & $2.40 \times 10^{-200}$ \\
Breast cancer & 3,180 & 380 & $2.47 \times 10^{-195}$ & $2.71 \times 10^{-197}$ \\
Colorectal cancer & 2,326 & 338 & $1.39 \times 10^{-194}$ & $1.67 \times 10^{-196}$ \\
Hepatocellular carcinoma & 2,450 & 337 & $6.40 \times 10^{-186}$ & $1.02 \times 10^{-187}$ \\
Melanoma & 1,398 & 272 & $5.08 \times 10^{-183}$ & $8.63 \times 10^{-185}$ \\
Lung cancer & 1,745 & 294 & $5.29 \times 10^{-182}$ & $9.53 \times 10^{-184}$ \\
\hline
\end{tabular}

The P-value for each sub-network in the table was calculated using the one-tailed Fisher's exact test against the hypothesis that a randomly selected gene set of the same size (579) could present an equal or higher number of overlapped genes with the corresponding gene sub-network. All these sub-networks passed FDR correction $(\mathrm{q}=0.005)$. FDR, false discovery rate.

results indicated that each of the four scores proposed in this study was effective in selecting top genes for CML prediction and that these identified genes may lead to an improved classification accuracy. Therefore, top genes that were present in all four scores may be considered as significant CML target genes.
In Fig. 3, the inner circle depicts the top genes selected using SScore. The size of the circle is directly proportional to the PScore and the brightness in color corresponds to the RScore. According to the aforementioned hypothesis, genes with high SScore, PScore and RScore values should be considered as 


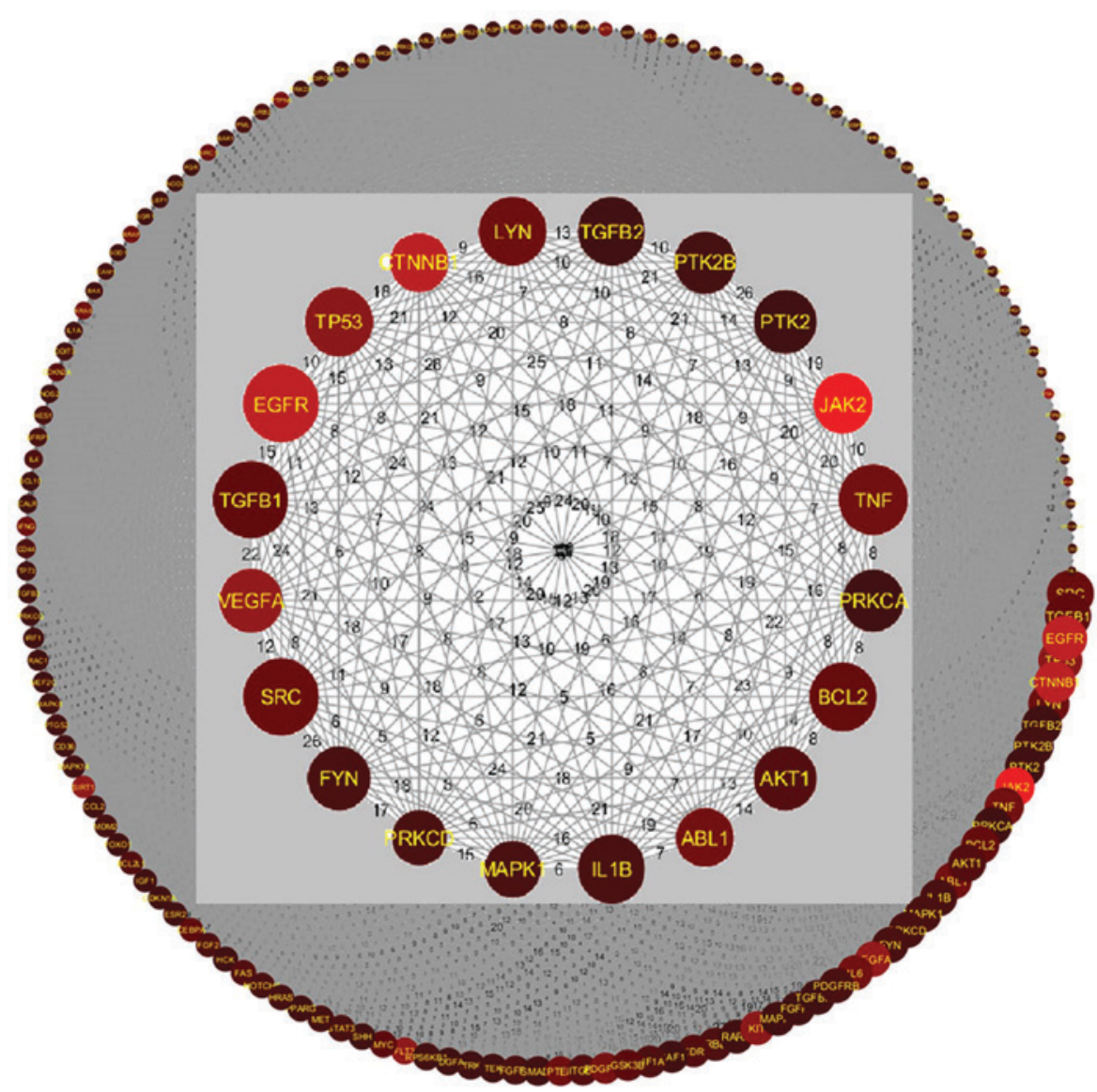

Figure 3. Pathway-based GGI network. The outer circle contains all 485 out of 579 genes and the inner circle contains the top 20 genes determined by the SScore. The size and brightness of the circle is directly proportional to the PScore and RScore, respectively. The number between two genes represents the number of shared pathways. GGI, gene-gene interaction; PScore, pathway score; RScore, reference score; SScore, network significance score.

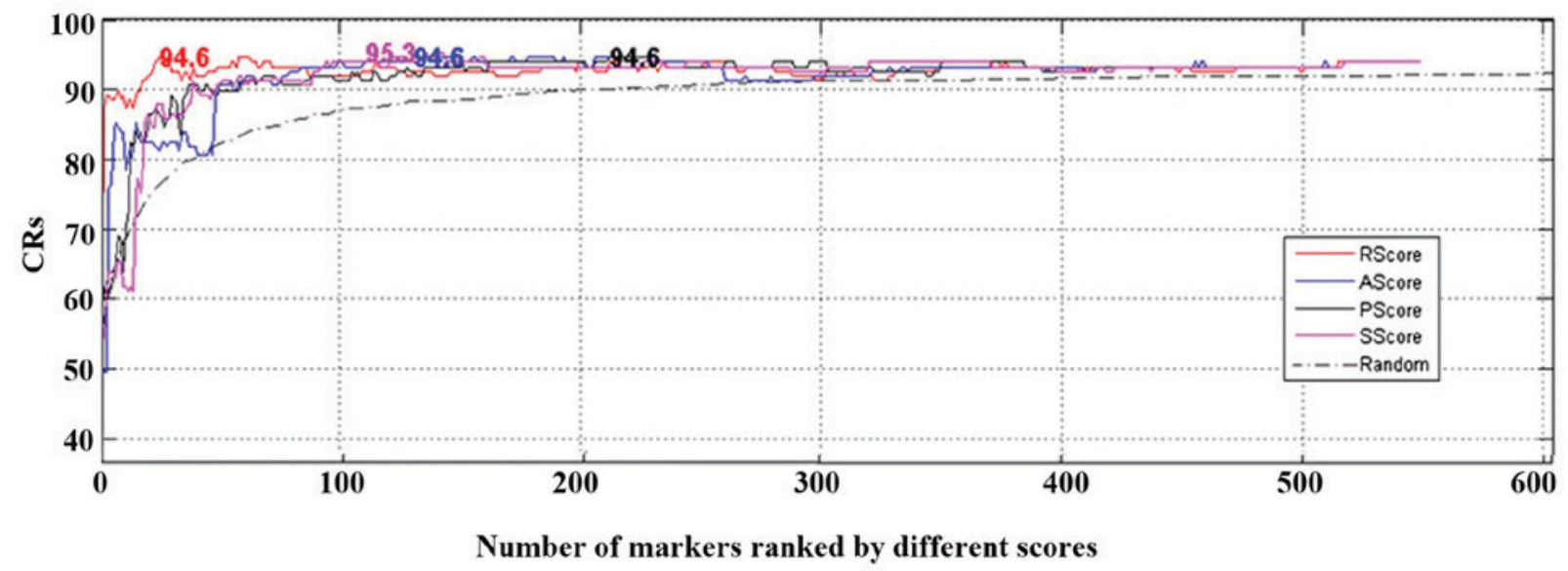

Figure 4. Classification results of genes ranked using different metrics. The numbers indicate the maximum CR achieved using different scores. The 'Random' line was generated using 5,000 permutations and represents the mean CR when randomly selecting a gene set with $n(n=1,2 \ldots)$ number of genes. AScore, age score; CR, classification accuracy; PScore, pathway score; RScore, reference score; SScore, network significance score.

important for CML. The relevant genes were EGFR, TP53, CTNNB1, JAK2, TNF, ABL1, VEGFA, B-cell lymphoma 2 (BCL2) and SRC proto-oncogene (SRC), which are presented in Fig. 3 as large, bright red circles. Analysis demonstrated that on average these genes have an RScore $=22.22 \pm 11.76$, PScore $=38.67 \pm 3.74$ and SScore $=2.54 \pm 0.16$. Please refer to CML_GD $\rightarrow$ Related Genes for further information.
Some genes exhibited a high PScore and SScore, but low RScore; these included SRC, transforming growth factor $\beta 1$ (TGFB1), lck/yes-related novel protein tyrosine kinase, TGFB2, protein tyrosine kinase $2 \beta$ (PTK2B), PTK2, protein kinase c alpha, BCL2, AKT serine/threonine kinase 1, IL1B, mitogen-activated protein kinase 1 (MAPK1), FYN proto-oncogene, platelet derived growth factor receptor $\beta$ 
Table III. Permutation test on top genes corresponding to highest CRs.

\begin{tabular}{lccccc}
\hline Variable & RScore & AScore & PScore & SScore & 549 genes \\
\hline Max CRs (\%) & 94.63 & 94.63 & 94.63 & 95.30 & 93.96 \\
No. of genes & 25 & 131 & 212 & 111 & 549 \\
P-value & $<2 \times 10^{-4}$ & 0.0124 & 0.029 & 0.0008 & 0.215 \\
\hline
\end{tabular}

P-value represents the permutation P-value, which is defined by the number of runs with equal or higher CRs divided by the total number of runs. AScore, age score; CR, classification accuracy; PScore, pathway score; RScore, reference score; SScore, network significance score.

and MAPK3 (Fig. 3; CML_GD $\rightarrow$ Related Genes). These genes were present in multiple CML-implicated pathways (PScore=39.21 \pm 3.70$)$ and displayed strong connections with other CML target genes (SScore $=2.55 \pm 0.16$ ); however, these genes were first reported many years ago (AScore $=14.00 \pm 7.18)$ with few references available (RScore $=4.36 \pm 3.61$ ). These findings indicated that further research on these genes may improve the understanding of their true relevance for CML. In addition, the 'inner circle' can be increased to include more genes with less significance for future analysis.

\section{Discussion}

Besides BCR-ABL mutations, many other genes have been associated with CML (8-14). However, due to the inaccuracies introduced during subject recruitment, data collection and processing, these findings require further validation. In the present study, literature-based CML-gene relation data and gene expression data, together with enrichment analysis and GGI analysis were employed to study pathogenic interactions between 579 target genes and CML. Four metrics were proposed and evaluated using a case/control classification approach. The results demonstrated that most of the CML target genes interacted with each other and served a role within numerous CML-implicated pathways.

A total of 579 genes were collected and evaluated, as these were supported by 1,739 references to demonstrate association with CML (CML_GD $\rightarrow$ Ref for Disease-Gene Relation). Both GSEA and SNEA were conducted to analyze the functional roles of these genes in the pathogenesis of CML. The GSEA results demonstrated that 485 out of 579 genes were significantly enriched in 145 pathways $\left(\mathrm{P}<8.2 \times 10^{-11} ; \mathrm{q}=0.005\right)$. Many of these pathways have been previously implicated in CML $\left(C M L \_G D \rightarrow\right.$ Related Pathways), including three nervous system, three immune system and two ageing-related pathways (28-36). Furthermore, GGI analysis revealed that most CML candidate genes exhibited a strong functional association with each other. Notably, $>70 \%$ of the 579 genes were also associated with many other diseases, including acute myeloid leukemia, myelodysplastic syndrome and lymphoma (CML_ $\mathrm{GD} \rightarrow$ Related Diseases), which are linked to CML (37-39).

In the present study, four metrics were proposed to select target genes relevant for CML. RScore was used to identify genes that have been frequently reported to be associated with
CML, PScore was used to find genes linked to CML-related pathways, SScore was used to discover genes with a strong functional association with other CML candidate genes and AScore was used to identify newly discovered CML genes.

The results from the case/control classification approach demonstrated the effectiveness of the proposed metrics. In particular, genes selected by the four metric scores led to effective CML case/control prediction ( $\mathrm{CRs}>94 \%$; $\mathrm{P}<0.029)$, whereas non-effective predication accuracy $(\mathrm{P}=0.215)$ was achieved when all the target genes were included in the dataset (GSE13159; 549 out of 579 genes). These results suggested the necessity of using node (gene) metrics for further analysis of the CML candidate genes.

Cross-network metrics analysis revealed nine top CML candidate target genes, including EGFR, TP53, CTNNB1, JAK2, TNF, ABL1, VEGFA, BCL2 and SRC. These genes have been reported by numerous independent studies for their association with CML (RScore=22.22 \pm 11.76$)(7-14)$, have a role within CML candidate pathways $(\mathrm{PScore}=38.67 \pm 3.74$ ) and display strong functional linkage with other CML candidate genes (SScore $=2.54 \pm 0.16$ ). Therefore, the results demonstrated that these genes may be associated with a higher probability of developing CML.

Although this study evaluated 579 known CML candidate genes acquired from CML-gene relation data, the proposed PScore and SScore can be used to evaluate any gene included in the CML pathways. In addition, more/novel genes can be evaluated following the same approach. Further metrics may also be tested for the evaluation of CML target genes. Lastly, the results presented in this study are computational rather than experimental; therefore, biological experiments are required to test the functional mechanism of these significant CML genes.

In conclusion, to the best of our knowledge, this study has demonstrated for the first time integration of large-scale literature-based relation data and gene expression data, together with enrichment analysis and GGI analysis to evaluate 579 genes for their association with CML. The proposed computational approach utilized a weighted network that may help identification of significant or novel CML target genes for future research.

\section{Acknowledgements}

Not applicable.

\section{Funding}

The present study was supported by the Program for the National Natural Sciences Foundation of China (grant no. 81500162), the Shanghai Three-Year Plan of the Key Subjects Construction (grant nos. 15GWZK0102 and 16CR1034B).

\section{Availability of data and materials}

All data generated or analyzed during this study are included in this published article.

\section{Authors' contributions}

YZ, YW, HC and XZ contributed to the study design and the writing of the manuscript; $\mathrm{YZ}$ and $\mathrm{HC}$ made substantial 
contributions to data analysis. YW and QC made substantial contributions to the concept and design of the present study. All authors read and approved the final manuscript.

\section{Ethics approval and consent to participate}

Not applicable.

\section{Consent for publication}

Not applicable.

\section{Competing interests}

The authors declare that they have no competing interests.

\section{References}

1. Garcia-Manero G, Faderl S, O'Brien S, Cortes J, Talpaz M and Kantarjian HM: Chronic myelogenous leukemia: A review and update of therapeutic strategies. Cancer 98: 437-57, 2003.

2. Kantarjian HM, Giles FJ, O'Brien SM and Talpaz M: Clinical course and therapy of chronic myelogenous leukemia with interferon-alpha and chemotherapy. Hematol Oncol Clin North Am 12: 31-80, 1998.

3. Nowell PC: Discovery of the Philadelphia chromosome: A personal perspective. J Clin Invest 117: 2033-2035, 2007.

4. De Bono JS and Ashworth A: Translating cancer research into targeted therapeutics. Nature 467: 543-549, 2010.

5. Yogalingam G and Pendergast AM: Abl kinases regulate autophagy by promoting the trafficking and function of lysosomal components. J Biol Chem 283: 35941-35953, 2008.

6. Daniel L, Ahmed CM, Bloodgood RS, Kidd JR, Castiglione CM Duttagupta S and Lebowitz P: Polymorphism of the human c-abl gene: Relation to incidence and course of chronic myelogenous leukemia. Oncogene 1: 193-200, 1987.

7. Anand M, Chodda SK, Parikh PM and Nadkarni JS: Abnormal levels of proinflammatory cytokines in serum and monocyte cultures from patients with chronic myeloid leukemia in different stages, and their role in prognosis. Hematol Oncol 16: 143-154, 1998.

8. Singer MK, Assem M, Abdel Ghaffar AB and Morcos NY: Cytokine profiling as a prognostic markers in chronic myeloid leukemia patients. Egyp J Immunol 18: 37-46, 2011.

9. Li L, Xu N, Zhang JF, Xu LL, Zhou X, Huang BT, Li YL and Liu XL: Ephb4/ephrinb2 contributes to imatinib resistance in chronic myeloid leukemia involved in cytoskeletal proteins. Int J Med Sci 13: 365-373, 2016.

10. Tefferi A, Thiele J, Vannucchi AM and Barbui T: An overview on CALR and CSF3R mutations and a proposal for revision of WHO diagnostic criteria for myeloproliferative neoplasms. Leukemia 28: 1407-1413, 2014.

11. Perez CA, Velez M, Raez LE and Santos ES: Overcoming the resistance to crizotinib in patients with non-small cell lung cancer harboring EML4/ALK translocation. Lung Cancer 84: 110-115, 2014.

12. McCubrey JA, Steelman LS, Bertrand FE, Davis NM, Abrams SL, Montalto G, D'Assoro AB, Libra M, Nicoletti F, Maestro R, et al: Multifaceted roles of GSK-3 and Wnt/ $\beta$-catenin in hematopoiesis and leukemogenesis: Opportunities for therapeutic intervention. Leukemia 28: 15-33, 2014.

13. Ouerhani S, Gharbi H, Menif S, Safra I, Douzi K and Abbes S: KIT mutation detection in Tunisian patients with newly diagnosed myelogenous leukemia: Prevalence and prognostic significance. Cancer Genet 205: 436-41, 2012.

14. Hoermann G, Greiner G and Valent P: Cytokine regulation of microenvironmental cells in myeloproliferative neoplasms. Mediators Inflamm 2015: 869242, 2015.

15. Nikitin A, Egorov S, Daraselia N and Mazo I: Pathway studio-the analysis and navigation of molecular networks. Bioinformatics 19: 2155-2157, 2003.

16. Abdelmoez A, Coraça-Huber DC, Thurner GC, Debbage P, Lukas P, Skvortsov S and Skvortsova II: Screening and identification of molecular targets for cancer therapy. Cancer Lett 387: 3-9, 2017.
17. Li J, Sheng N, Cui R, Feng Y, Shao B, Guo X, Zhang H and Dai J: Gestational and lactational exposure to bisphenol AF in maternal rats increases testosterone levels in 23-day-old male offspring. Chemosphere 163: 552-561, 2016.

18. Cao H, Duan J, Lin D, Shugart YY, Calhoun V and Wang YP: Sparse representation based biomarker selection for schizophrenia with integrated analysis of fMRI and SNPs. Neuroimage 102 Pt 1: 220-228, 2014.

19. Daraselia N, Yuryev A, Egorov S, Novichkova S, Nikitin A and Mazo I: Extracting human protein interactions from MEDLINE using a full-sentence parser. Bioinformatics 20: 604-611, 2004.

20. Lorenzi PL, Claerhout S, Mills GB and Weinstein JN: A curated census of autophagy-modulating proteins and small molecules: Candidate targets for cancer therapy. Autophagy 10: 1316-1326, 2014.

21. Sivachenko AY, Yuryev A, Daraselia N and Mazo I: Molecular networks in microarray analysis. J Bioinform Comput Biol 5: 429-456, 2007.

22. Benjamini Y and Hochberg Y: Controlling the false discovery rate: A practical and powerful approach to multiple testing. J Royal Statistical Society, Series B (Methodological) 57: 289-300, 1995.

23. Opsahl T, Agneessens F and Skvoretz J: Node centrality in weighted networks: Generalizing degree and shortest paths. Social Networks 32: 245-251, 2010.

24. Freeman LC: Centrality in social networks: Conceptual clarification. Social Networks 1: 215-239, 1978.

25. Yang S and Knoke D: Optimal connections: Strength and distance in valued graphs. Social Networks 23: 285-295, 2001.

26. Wang J, Cao H, Liao Y, Liu W, Tan L, Tang Y, Chen J, Xu X, Li H, Luo C, et al: Three dysconnectivity patterns in treatment-resistant schizophrenia patients and their unaffected siblings. Neuroimage Clin 8: 95-103, 2015.

27. Hehlmann R, Hochhaus A and Baccarani M; European Leukemia Net: Chronic myeloid leukaemia. Lancet 370: 342-350, 2007.

28. Gong IY and Kim RB: Impact of genetic variation in OATP transporters to drug disposition and response. Drug Metab Pharmacokinet 28: 4-18, 2013.

29. Sawyers CL, McLaughlin J and Witte ON: Genetic requirement for Ras in the transformation of fibroblasts and hematopoietic cells by the Bcr-Abl oncogene. J Exp Med 181: 307-313, 1995.

30. Rahmani M, Nguyen TK, Dent P and Grant S: The multikinase inhibitor sorafenib induces apoptosis in highly imatinib mesylate-resistant BCR/ABL+ human leukemia cells in association with STAT5 inhibition and MCL-1 down-regulation. Mol Pharmacol 72: 788-795, 2007.

31. Bi S, Barton CM, Lemoine NR, Cross NC and Goldman JM: Retroviral transduction of Philadelphia-positive chronic myeloid leukemia cells with a human mutant p53 cDNA and its effect on in vitro proliferation. Exp Hematol 22: 95-99, 1994.

32. Clark RE: Immunotherapeutic strategies in chronic myeloid leukemia. Curr Hematol Malig Rep 2: 89-94, 2007.

33. Pendergast AM, Gishizky ML, Havlik MH and Witte ON: SH1 domain autophosphorylation of $\mathrm{P} 210 \mathrm{BCR} / \mathrm{ABL}$ is required for transformation but not growth factor independence. Mol Cell Biol 13: 1728-1736, 1993.

34. Lugo TG, Pendergast AM, Muller AJ and Witte ON: Tyrosine kinase activity and transformation potency of bcr-abl oncogene products. Science 247: 1079-1082, 1990.

35. Waight JD, Banik D, Griffiths EA, Nemeth MJ and Abrams SI: Regulation of the interferon regulatory factor-8 (IRF-8) tumor suppressor gene by the signal transducer and activator of transcription 5 (STAT5) transcription factor in chronic myeloid leukemia. J Biol Chem 289: 15642-15652, 2014.

36. Rossi DJ, Jamieson CH and Weissman IL: Stems cells and the pathways to aging and cancer. Cell 132: 681-696, 2008.

37. Pawarode A, Sait SN, Nganga A, Coignet LJ, Barcos M and Baer MR: Acute myeloid leukemia developing during imatinib mesylate therapy for chronic myeloid leukemia in the absence of new cytogenetic abnormalities. Leuk Res 31: 1589-1592, 2007.

38. Kovitz C, Kantarjian H, Garcia-Manero G, Abruzzo LV and Cortes J: Myelodysplastic syndromes and acute leukemia developing after imatinib mesylate therapy for chronic myeloid leukemia. Blood 108: 2811-2813, 2006.

39. Găman AM, Dobrea C and Rotaru I: A case of non-Hodgkin lymphoma in a patient with chronic myeloid leukemia. Rom J Morphol Embryol 54: 1141-1145, 2013. 